\title{
Weak and Electromagnetic Interactions in Nuclei
}

\author{
Evolution of nuclear physics into the particle domain
}

\author{
H.V. Klapdor, Heidelberg
}

(Max-Planck-Institut für Kernphysik)

Nuclear physics is presently experiencing a new impetus, addressing itself towards more fundamental physics questions and becoming more and more important as a test of physics beyond the standard model of grand unification. The close connections between the laws of microphysics, astrophysics and cosmology - one of the most significant discoveries of our century - open further exciting perspectives.

Among the most topical fields of activity (see K. Gabathuler, Europhys. News, Jan. 1987) are the search for a finite neutrino mass by $\beta$-end-point spectroscopy, neutrinoless double beta decay and neutrino oscillations, proton decay, the study of rare and forbidden muon and pion decays and the search for an electric dipole moment of the neutron - all experiments which are complementary to the direct search for new particles and symmetries in high energy experiments - and their relations to grand unification, superstring theories and the evolution of the early Universe. Other topics which not so long ago would have been regarded exclusively as particle physics are the status of electroweak gauge theories, in particular the mixing of quarks by the weak interaction and the origin of the observed $\mathrm{CP}$ violation, quarks in nucleons and nuclei, etc. Even traditional fields of nuclear physics carry the same message: the discussions on the positron lines oberved at GSI in collisions of nuclei with high $\mathrm{Z}$; the observation of extremely high spins in super-deformed nuclei; new results on the parity violation in quasi-elastic electron scattering; properties of exotic nuclei and of nuclear matter. But this is now close to astrophysics - the collapse of heavy stars, baryon and lepton number conservation and weak interactions in cosmology.

Weak interactions are of basic importance for the development and structure of the Universe because:

- without weak interactions there would be no heavy elements;

- neutrinos could determine by their
This review of developments in nuclear physics is largely based on discussions at the EPS and IUPAP sponsored International Symposium on Weak and Electromagnetic Interactions in Nuclei, W.E.I.N. 1986, held in Heidelberg, 1-5 July 1986, in conjunction with the 600th anniversary of the University of Heidelberg. About 300 participants worldwide attended the Conference, which was held under the patronage of the Rector of the University, Gisbert zu Putlitz and chaired by the author. Several countries expressed their interest in hosting a similar W.E.I.N. conference in 1989. The Proceedings have been published recently by Springer-Verlag.

mass, whether the Universe is open or closed;

- neutrinos are the best candidates for the non-baryonic dark matter in the Universe, which could represent more than $90 \%$ of the mass of the cosmos;

- problems of the weak interaction, such as the mass of the neutrino, are together with the half-life of the proton - key points for the structure of grand unification theories (GUTs) or supersymmetry theories (SUSYs) which must include the development of the early Universe.

\section{Neutrinos}

Today, 30 years after the direct observation of the neutrino in the famous Goldhaber experiment, it is still unknown whether the neutrino has a mass or not, and all over the world there are different projects trying to resolve the question.

The European Gallex group (Gallium Experiment) headed by the MPI in Heidelberg plans to detect solar neutrinos in the Gran Sasso tunnel near Rome; R. Mössbauer and his group are looking for neutrino oscillations using the core of a nuclear power reactor as a neutrino source. Both these experiments are looking for differences in the masses of different neutrino types. Solar neutrino experiments with other detectors are expected to yield answers to the recent question of neutrino-matter oscillations.

Two other types of experiment attack the mass question directly. W. Kündig in Zürich studies the beta decay of tritium and has reported an upper limit of $18 \mathrm{eV}$. A different method is to measure the half-life for neutrinoless double beta decay and here the most active groups are in Osaka, Columbia, Milan, Zaragoza and Santa Barbara.

To deduce the neutrino mass from observations of the decay, additional nuclear structure information is needed, but using the most recent calculations of the nuclear matrix elements done in Heidelberg, the mass of the electron neutrino turns out to be less than $1 \mathrm{eV}$. This limit corresponds, however, to an effective mass only. If more than one Majorana neutrino couples to the electron, as the interference between the different mass states of the neutrinos in the neutrino exchange amplitude could be destructive, the true mass of the electron neutrino could be substantially larger. If one assumes a second neutrino with a mass not smaller than $100 \mathrm{MeV}$, a conservative limit for the electron neutrino mass is then $13 \mathrm{eV}$. Of particular interest are the Osaka group's studies of the $\mathrm{O}^{+} \rightarrow 2^{+}$transitions which in contrast to the $\mathrm{O}^{+} \rightarrow \mathrm{O}^{+}$ground state transitions, can be driven by right-handed currents only, not by a mass term, and further efforts to look for a neutrinoless decay branch with emission of a light boson, the majoron.

To reduce further the limit on the halflife for neutrinoless $\beta \beta$ decay by more than an order of magnitude, a collaboration from Heidelberg, Leningrad, Moscow, and Bordeaux is planning to use enriched ${ }^{76} \mathrm{Ge}$ inside a well-shielded cavity. The Soviet Union will provide the enriched material.

An important constraint for deducing 
the neutrino mass is provided by nuclear reaction measurements. In $(p, n)$ charge exchange reactions, $\beta^{-}$strength distributions have a control function not only for the calculation of the double beta matrix elements required for deducing the neutrino mass, but also for the determination of the efficiency of solar neutrino detectors such as the Gallium detector.

\section{Muon Physics}

The question of muon-to-electron conversion is as old as the muon itself. When it was realized that the muon does not decay into an electron and a photon, the concept of separately conserved muonic and electronic lepton numbers was introduced as an ad hoc hypothesis. Only recently has this question become a very important issue of particle physics. In some extensions of the standard model it leads to the most sensitive mass limit on new particles such as Higgs scalars, leptoquarks and heavy neutrinos.

The signature for coherent $\mu$-e conversion in which the nucleus is left in its ground state is muon capture accompanied by the emission of an electron at an energy $E_{\mathrm{e}} \approx m_{\mathrm{u}} c^{2}-B=104 \mathrm{MeV}$, where $B$ is the binding energy of the muonic atom. From the Canadian meson facility Triumf a preliminary upper limit has been reported for the branching ratio, $R$, of muon-to-electron conversion compared to total muon capture of $R<$ $4 \times 10^{-12}$ (90\% confidence limit).

The experimental results of the European Muon Collaboration (EMC) and other electron, muon and neutrino experiments concerning differences between the momentum distribution of quarks in the nucleus from that in free nucleons (EMC effect) are difficult to explain and new data are awaited from the New Muon Collaboration (NMC) at CERN. According to E. Berger from Argonne, the Drell-Yan process is expected to provide an answer, but there are various models on the market. A Russian-Danish-American collaboration believes that part of the EMC effect could be explained by simple binding effects. An alternative approach which leans on QCD models due to F. Close from the Rutherford Laboratory connects the modification to a change of the confinement scale in nuclei originating from an increase in the size of nucleons, the formation of multiquark clusters or of a large quark bag. This however clashes with the view held by I. Sick of Basel that the size of nucleons should be only a very little larger in nuclei than when free, if at all.
Tests on the Standard Theory, GUTs etc.

In the latest measurements reported on proton life-times, $\mathrm{H}$. Meyer from Wuppertal has given a lower limit for the half-life of the proton decaying into a neutral pion and a positron of $4 \times 10^{32}$ years. This result rules out the simplest GUT - the minimal SU5 model which predicts a half-life of less than 3 $\times 10^{31}$ years. Certain modifications to GUT and supersymmetry models, however, could remove this objection.

New measurements have been made of the electric dipole moment of the neutron. The purpose of the experiment is to test $\mathrm{CP}$ violation which is observed only in the decay of the neutral kaon. The latest value reported by N. Ramsey from Harvard and V. Lobashev from Moscow is $[-1.8 \pm 2.9] \times 10^{-25} \mathrm{~cm}$. (The smallness of the dipole moment can be pictured by enlarging the neutron to the size of the Earth and observing that the radius of the charge distribution at the pole would differ from that at the equator by only $0.01 \mathrm{~mm}$ !). Theoretical predictions range from $10^{-19}-10^{-32} \mathrm{~cm}$ and the new upper limit leaves roughly four categories of theory still within the experimental bounds: phenomenological milli-weak and super-weak theories, and gauge theories that attribute the $T$ violation to the mixing of six or more quarks, or to the exchange of multiple Higgs bosons.

Extensions of the standard model are seen by R. Peccei (Europhys. News, Jan. 1987) to link such apparently disconnected phenomena as small neutrino masses, visible (and invisible) axions, the $\mathrm{CP}$ problem and the Universe, and baryon asymmetry. The exciting possibility that the positron peaks observed in collisions of high $Z$ nuclei at GSI originate from the decay of an axion is ruled out by the low probability for rare and forbidden decays of the muon and $\pi$ meson. This result by R. Engfer and his group at SIN is confirmed by a search for short-lived axions by neutron capture on protons performed with the Grenoble cold neutron beam and also by a search for pseudoscalar axions in isoscalar $\mathrm{M} 1$ transitions in ${ }^{10} \mathrm{~B}$ in Amsterdam.

\section{Exotic Nuclei, Nuclear Matter and \\ Astrophysics}

New information is being produced on beta decay properties - half-lives, beta-delayed neutron emission and fission rates - which is of great importance for modelling theories that predict beta decay properties of nuclei not accessible to terrestrial laboratories. The latter enter sensitively into our under- standing and theoretical "reproduction" of element synthesis in the Universe, and of the age of our Galaxy as deduced from cosmochronometers.

Nuclear electron capture plays a decisive role at several stages in the evolution of massive stars. Electron capture by nuclei of the iron group in the core partly triggers the gravitational collapse of Type II supernova progenitors, once the Chandrasehkar limit has been reached. Also hydrodynamical shock propagation in the post-bound phase and "delayed" explosions depend on the neutrino luminosity behind the shock, which is governed by capture in the homologous core. The weak interaction also controls the life of a neutron star in its primary phase. The collapse scenario of a heavy star could be quite different from the standard collapse scenario which is a low-entropy collapse - in the case of lepton number violation, mediated e.g. by majorons.

Properties of nuclear matter play a significant part also in stellar evolution. A new equation of state for nuclear matter at high density has been used by $\mathrm{S}$. Kahana and collaborators from Brookhaven National Laboratory in hydrodynamical simulations of stellar collapse. These calculations are the first to produce a prompt shock-explosive mechanism for Type II supernovae using realistic evolutionary supernova models.

Over the years, two major classes of mechanism had been proposed: One approach sought an ejection of the stellar mantle and envelope by an explosive shock created in a core bounce after collapse. The second approach would have the exterior regions blown off by the energetic neutrinos somewhat after collapse. Both approaches failed however, to give the desired effect even after correction for the initial omission of important beta-capture channels, which led to a reduction of the core mass.

The new break-through is achieved by theoretically altering the experimentally rather uncertain equation of state for nuclear matter at high density in the direction of softening, and introducing relativistic gravitation into the collapse phase. Both lead to an increase of the energy initially pumped into the shock. This result is important also for understanding the synthesis of heavy elements in the Universe by the $r$ (rapid neutron capture) process in explosive helium-burning.

\section{Weak Interactions and Cosmology}

In most current evolutionary models of the Universe only $10 \%$ of its mass consists of nucleons. Candidates for the 
dark matter fall into two categories: hot (neutrino-like) and cold (axion- and photino-like) particles, the first being the more probable solution. The necessity for accommodating non-baryonic dark matter, the recently discovered largescale structure of the Universe (many large voids with most galaxies distributed on the walls of the voids - and not randomly!) and the early origin of the galaxies together set strict boundary conditions for cosmological models.

Although there is not yet a fully consistent picture of the early Universe, a phase of inflationary expansion in the early cosmos is believed by some (see e.g. J. Ellis, Europhys. News, Sept. 1985) to offer the most natural explanation for the isotropy of the microwave background radiation, the flatness of space (i.e., its Euclidean metric) and the elimination of "unwanted" particles like monopoles, gravitinos, etc. There remains, however, the problem of the cosmological constant, which has on the one hand to be large to trigger the inflationary expansion, and, on the other, small in order not to contradict presentday cosmological observation. The cosmological constant introduced into the equations of general relativity in 1917 by Einstein to support a stationary Universe and removed by him in 1931, is nowadays a natural consequence of quantum field theory and corresponds to an energy density of the vacuum. A positive vacuum energy (cosmological constant) leads to an expansion pressure.

In principle a large energy density of the vacuum can be produced in the early Universe by the hypothetical Higgs fields which in our understanding are responsible for spontaneous symmetry breaking. A mechanism reducing the corresponding large $\Lambda$ to a value consistent with the present status of the Universe (i.e., by more than 50 orders of magnitude) is, however, not yet known.

Different views have been expressed over the aesthetical requirement for an exactly vanishing cosmological constant, but there seems to be increasing evidence that the age of the Universe is greater than $15 \times 10^{9}$ years. G. Tammann from Basel estimates $18 \pm 3 \times$ $10^{9}$ years from measurements on globular clusters, consistent with the recent Heidelberg figure of about $20 \times 10^{9}$ years from cosmochronometers. This would indicate a non-vanishing cosmological constant for values of the Hubble constant $H_{0} \geq 45 \mathrm{~km} \mathrm{Mpc}^{-1} \mathrm{~s}^{-1}$ which by observation is currently constrained to values between around 40 and $100 \mathrm{~km}$ $\mathrm{Mpc}^{-1} \mathrm{~s}^{-1}$.

\section{Record Nuclear Spins}

Despite its extensions into particles at one end of the scale and cosmology at the other, nuclear physics has nevertheless its own record book still. J. Sharpey-Schafer and his group from the University of Liverpool have reported the measurement of the highest spin seen in an atomic nucleus, notably $60 \hbar-$ about 20 units higher than previously. This was discovered in a super-deformed rotational band of ${ }^{152} \mathrm{Dy}$ using a detector system (TESSA III) which allows spectroscopy of a new class of superdeformed prolate nuclei whose major to minor axes have a ratio of 2:1.

Am Physikalischen Institut der Universität Bern ist die vollamtliche

\section{Professur für experimentelle Elementarteilchenphysik}

neu zu besetzen.

Die Bewerber müssen sich über grundlegende und originelle Beiträge zur Teilchen- und Hochenergiephysik ausweisen können. Sie sollten befähigt sein, die Leitung der laufenden Forschung auf dem Gebiete der Teilchenphysik mit Beschleunigern und der Astroteilchenphysik zu übernehmen und neue Forschungsprojekte zu initiieren und zu leiten.

Der Aufgabenbereich umfasst die Leitung des Laboratoriums für Hochenergiephysik, dem ein qualifizierter Dozenten- und Mitarbeiterstab angehört, die Mitwirkung im Unterricht für Anfänger verschiedener Fachrichtungen und für fortgeschrittene Physikstudenten, die Betreuung von Lizentianden und Doktoranden, sowie die Erledigung allgemeiner Institutsund Fakultätsgeschäfte.

Weitere Angaben über die offene Professur sind bei Prof. J. Geiss, Physikalisches Institut, Sidlerstrasse 5, CH-3012 Bern, erhältlich.

Bewerbungen mit curriculum vitae, Publikationsverzeichnis, Angaben über die Forschungsabsichten und Referenzen sind bis 1. Mai 1987 an die Erziehungsdirektion des Kantons Bern, Abteilung Hochschulwesen, Sulgeneckstrasse 70, CH-3005 Bern, zu richten.

\section{Adam Hilger Introduction to Gauge Field Theory \\ David Bailin, University of Sussex and Alexander Love, University of London \\ Graduate Student Series in Physics Series editor Professor D F Brewer, University of Sussex}

An introduction to gauge field theory for graduate students, advanced undergraduates and researchers in the field of particle physics. Assuming a knowledge of relativistic quantum mechanics, but not of quantum field theory, the book approaches the subject entirely from a path integral standpoint without any reliance on the more traditional method of canonical quantisation. The topics covered form a foundation for a knowledge of modern relativistic quantum field theory.

$1986 \quad 234 \times 156 \mathrm{~mm} \quad 362$ pages illustrated hardcover $0852748175 \mathrm{E} 38.00$ paperback 0852748183 £ 19.50

Gauge Theories

in Particle Physics

I J R Aitchison, University of Oxford and A J G Hey,

University of Southampton

Graduate Student Series in Physics

Series editor Professor D F Brewer, University of Sussex

An intuitive approach to the ideas and methods of gauge field theories in elementary particle physics. The authors have dispensed with the formalism of quantum field theory to present these fundamental developments at a level which is accessible to new graduate students, experimental workers and others without a theoretical background in the subject.

$1982 \quad 234 \times 156 \mathrm{~mm} \quad 357$ pages illustrated paperback $0852745346 \mathrm{f} 14.50$

\section{Standard Model and Beyond}

Edited by W J Zakrzewski, University of Durham

Consultant editor Professor E J Squires, University of Durham

Theoretical high energy physics continues to develop rapidly. This book, based on six lectures given at a Summer School in Theoretical Elementary Particle Physics in September at Durham University provides a pedagogical review of currently interesting topics in high energy physics. Intended for postgraduate students just completing their first year of research, it will also be of interest to researchers in this area.

May $1987234 \times 156 \mathrm{~mm} \quad$ c240 pages illustrated hardcover $085274367 \times$ c£ 20.00

For further details of these and other titles in high energy physics, please write to the publishers at the address below

\section{Adam Hilger}

Techno House, Redcliffe Way. Bristol BS1 6NX, UK Telephone: 0272276693 Telex: 449149 Exclusive North American distributors Taylor and Francis

242 Cherry Street, Philadelphia. PA 19106-1906, USA Telephone: (215) 2380939 Telex: 244489 